\title{
Implicit Gender-biased Speeches in Workplace: Embedded Stereotypes and Effects on Women's Career Development
}

\author{
Yijia Chai \\ ${ }^{1}$ Faculty of Humanities and Social Science, University of Newcastle upon Tyne, Newcastle, UK \\ *Corresponding author.Email: charlottechai@m.poe.edu.pl
}

\begin{abstract}
Women's career path still meets with many challenges because of sexism, which is regarded as a special case of ethnic prejudice. Based on the nature and sources of sexism, it is worth highlighting that the root of sexism is underpinned by a set of traditional gender stereotypes toward women. Stereotypes make claims about generalized and essentialized features about social kinds. They are cognitively embedded in people's daily speeches on occupational occasions and sometimes expressed in the form of generics. The author terms and analyzes two typical types of speech in occupational occasions: stereotyped questions in occupational contexts and benevolent speeches, inclusive of showing warmth toward women and giving praises. The analysis is done from the perspective of linguistics, psychology, and philosophy of language. Through the discussion, this paper argues that stereotypes covertly and in most of the case, unconsciously embedded in speeches expressed toward women in occupational contexts. Moreover, based on the analysis, the paper shows the adverse effects stereotypes have on women's career development. Conclusively, the stereotypes embedded in occupational speeches potentially reduce women's job opportunities and undermine their conceptions of working abilities.
\end{abstract}

Keywords: Stereotypes, Stereotyped speech, Generics, Women's career, Benevolent sexism

\section{INTRODUCTION}

Career development is still challenging for women in different professions across cultures because of sexism. For instance, there was a special column of sexist media coverage during the 2016 Rio Olympics on Mashable. One of its news blogs discussed a report about how languages create gendered attitudes. The report displayed that the frequent words used to portray female athletes "include 'pregnant,' 'married,' 'unmarried,' 'aged' and 'older'," none of which overlapped with words associated with men ("fast," "strong," "great," "big"). Fisk and Glick view sexism as a special form of prejudice characterized as showing ambivalent orientations toward women [1]. Among the typical factors that trigger this special form of prejudice, one of the most influential factors is stereotypes which exist in people's minds and are expressed via language in daily life. Specifically, stereotypes are sentences expressed in a unique linguistic form termed generics (this concept will be discussed in detail in Section 2) by linguistics to express essentialized generalizations [2]. As language is the "primary medium of social life" that undergirds thoughts and social practices, it is inevitable to focus on analyzing linguistic forms and their effects on society [3].

Although taking the form of generics, stereotypes are specifically referred to claims that are used to generalize and feature social groups, according to, for example, gender, race, or sexual orientation in different social and cultural milieus [2]. Nevertheless, unlike stereotypes embedded in hostile sexism that are expressed explicitly, stereotypes in the workplace are often expressed implicitly, unconsciously and even subjectively positively as benevolent sexism toward women. Notably, the phenomenon appears in different cultures and countries [4]. Given the detrimental effects and the prevalence of gender stereotypes, discussions are necessary on how they are embedded in speech covertly and their effects on women's career development. 
This paper aims to analyze stereotypes embedded in gender-biased speeches toward women in different occupational contexts and the potential adverse effects on women's career development. It is worth noting that the occupational contexts from which the examples were collected are defined in a broad sense, ranging from questions for female medalists in press conferences during the Tokyo 2020 Olympics, interviews for actresses after movie premieres, to speeches in ordinary workplaces. The author deduces two types of speech and calls them stereotyped questions in occupational contexts and benevolent speeches, inclusive of showing warmth toward women and giving praises. The two types of speech are made by speakers based on a specific property which is stereotypically regarded as an essence of the membership in the social category of women. The debate is framed through the lenses of philosophy of language, linguistics, and psychology. Section 2 will use previous studies on generics, stereotypes, and benevolent sexism to explore their relationships and display why gender-biased speeches in the workplace are worth discussing. Then, in section 3 , an analysis of stereotypes in gender-biased speeches will be displayed. In section 4, the author argues that the stereotypes in question, as a kind of generics, are detrimental to women's occupational path. Finally, section 5 contains a brief conclusion.

\section{GENERICS, STEREOTYPES AND BENEVOLENT SEXISM}

\subsection{Stereotypes as Essentialized Generalizations of Social Kinds}

Stereotypes, expressed in the form of generics, "can be characterized as a sub-class of generics, that make claims, specifically, about social kinds" [2]. Therefore, it is crucial and necessary to initially explore how generics influence people's cognition and language in a broader sense. Generics are sentences expressing generalizations without quantifiers like "most," "all," and "some." They make claims about natural and social groups. "Sharks attack swimmers," "women are submissive," and "doctors wear white coats" are examples of generic sentences. Research of linguists and philosophers on generics goes back to Lawler [5]. Since then, a considerable body of research has been done to discuss the categorization of generics, its linguistic forms, and the most complicated problem, the truth conditions for generics [2][6][7].

An important statement of generics is that generics could be default generalizations cognitively made by humans [8][9]. The statement offers one potential explanation about why generics exists commonly in different cultural and social milieus. Crucially, extensive empirical studies have demonstrated the hypothesis is probably true for firstly, young children are able to adopt and understand generic generalizations easily; secondly, both children and adults (in some specific contexts) would even interpret quantified generalizations as generics, as this is an easier way to understand the information. In light of this, stereotypes as a prominent kind of generics could play a significant role as cultural input for individuals through daily speeches in social contexts.

Psychologists introduce the term essentialist beliefs, or say generic beliefs, into the realm of generics and stereotypes. Psychological experimental evidence proves that generic beliefs play a decisive role in forming stereotype structures. Another important conclusion stemming from the related research is that there is a tendency for people to essentialize social kinds because "people believe that members of these categories share a fundamental nature that grounds a range of common properties" [8]. Gender categories, in this study, are one of the social categories that are commonly essentialized with deep, stable and enduring features [13]. Furthermore, people believe the essences of a social category are innate and inherent. Therefore, they tend not only to postulate that individuals of a social category share the essences in given social information, but also to "accommodate their incomplete representations of gender categories to novel social information." Fiske and Tylor also suggest that stereotypes basically function in two means: categorizing individuals based on their membership in a social category (such as gender, race, sexual orientations) and forming expectations of them [14].

\subsection{Stereotypes and Prejudice}

Notably, in his research on essentialist beliefs, Haslam reports that social categories which are essentialized with stable and enduring features are more likely to be treated in a demeaning and pernicious way [15]. In other words, there is a high possibility that essentialized social categories will be prejudiced. Similarly, it should be highlighted that stereotypes are typically interrelated with prejudice (of which sexism is a typical type) by both psychologists and linguists [16][17]. A considerable body of research has been done in reporting the relationship between stereotypes and prejudice. Mainly, stereotypes are stated as a crucial component of both prejudice attitudes and the process of prejudicing.

Intriguingly, although stereotypes are related to prejudice, which is naturally regarded as a set of negative attitudes toward social kinds [18], the question of why stereotypes are wrong remains puzzling. Theorists argue that by being stereotyped, people are deprived of their right to be acknowledged as individuals, which is disrespect for individuals [19]. However, Blum's argument is partly disagreed by 
Beeghly, who claims the view is "too strong." Beeghly suggests that stereotypes under varied circumstances are "epistemically and morally permissible" [2].

\subsection{Benevolent Sexism and Stereotypes}

Ambivalent sexism theory proposes two interrelated sexist attitudes toward women: hostile sexism (HS) and benevolent sexism (BS) [1]. Whereas hostile attitudes are obviously antagonistic and typically considered as what sexism is, generally including negative stereotypes and evaluations of women [16][1][4], benevolent sexism seems more implicit and unconsciously expressed in daily speech. Benevolent attitudes that are sexist "in terms of viewing women stereotypically and in restricted roles but that are subjectively positive in feeling tone" [1][20]. In a male-dominated workplace situation, for instance, a benevolent sexist leader would avoid assigning sophisticated tasks to a female employee, as the leader stereotypically believes the female employee should be taken care of by her male colleagues because of her membership of gender. Seemingly to his recipient(s), the leader shows a positive attitude toward women, but traditional gender stereotypes and the ideology of male dominance are underpinned in his intention. Glick and Fiske suggest that benevolent sexism is a type of prejudice, by all means, taking into consideration stereotypes and ideology it carries [1][21].

It is important to discuss BS in occupational spheres, for BS has been empirically proved as casually detrimental to women's professional competence by Dardenne et al. and Dumont and his colleagues [22][23]. The phenomenon is called positive stereotype threat [8][24]. Furthermore, Hidge and Ferris report that benevolent sexism subtly undermines gender equality in the workplace [4]. Apart from these influences on women's career development, subjectively positive attitudes, namely benevolent sexism, should be highlighted in view of its subtleness. Indeed, in modern occupational spheres, sexism is usually displayed in more covert and subtle ways and in a positive feeling tone, and thus, in some cases, people who express benevolent sexism "are less likely to be recognized as holding sexist views than when expressing hostile sexism" [25].

With a significant amount of previous research in generics, stereotypes and benevolent sexism, there still remains some gap for practically analyzing stereotypes embedded in speeches in the real-world workplace. The present study aims to do this kind of analysis from the perspective of linguistics and discuss the potential negative effects the speeches will have under the guidance of theories of stereotypes and benevolent sexism.

\section{ANALYSIS OF IMPLICIT GENDER-BIASED SPEECHES IN WORKPLACE}

\subsection{Stereotyped Occupational Questions}

In the current study, stereotyped questions in occupational contexts refer to questions about women's jobs or questions proposed in occupation-related contexts that are embedded with social stereotypes about women. Most importantly, these questions usually are not proposed to men in similar contexts. Examples are displayed in Table 1.

Table 1 Examples for Stereotyped Questions in occupational contexts

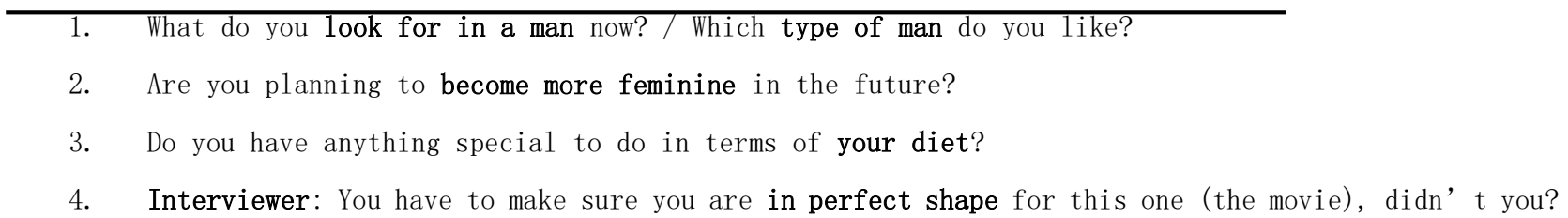

Actress: emmmm...

Interviewer: Is there any schedule you put yourself through in terms of the diet, the workout?

Q1 is a type of question typically asked for females in varied professions. The author collects these examples from two very different contexts: a press conference for several Chinese female athletes after they won first place in their respective fields and for a famous American female singer in an award ceremony. The questions, nevertheless, share a striking similarity with each other. This type of question is embedded with the stereotypes that can be expressed in generic sentences like 'women need boyfriends (romantic relations)' and 'women are vulnerable'. Questions like Q1 specifically imply that even if a woman achieves great success in her profession, such as winning the Olympic gold medal, she is still viewed through a stereotype lens and viewed as vulnerable and delicate. Therefore, she stereotypically necessarily needs a male to protect or take care of her. The thoughts derive from the ideology of paternalism, which believes that women are dependent on men playing roles as protectors and providers [1]. Notably, Q2 is a question for a Chinese female athlete of shot put. She has a strong body and a short hairstyle, which makes her appearance looks like a stereotypical male figure. Obviously, her body shape and the strength it brings are essential for shot put. 
However, generic generalizations essentialize what a woman's appearance traditionally looks like in society. Furthermore, the phenomenon also implicates there is indeed an "idealized" figure for women in social and cultural contexts [4]. Consequently, ignoring the female medalist, achievement, the interviewer stereotypes the woman based on her membership of a group, instead of treating the person as an individual.

Similarly, Qs 3-4 also implicate that having a good appearance is an essentialized feature for the social category of women. Stereotypes such as "women are in perfect body shapes," "women are slim," and "women know how to keep fit" are embedded in the speeches. In most cases, instead of focusing on women's performances in terms of their expertise, too much attention is paid to their looks. Sometimes, it seems that good-looking property is the sole contribution a woman can make to a business, especially in a situation like Q4 presents. In that case, the actress expresses her refusal to the question about her body shape by uttering "emmmm ..." but is ignored by the interviewer with a paraphrasing of his earlier question. To some extent, focusing on women' s body shape in an occupational context suggests the interviewers do not take women' $\mathrm{s}$ profession and career achievements seriously [1][20].

\subsection{Benevolent Speeches (inclusive of showing warmth toward women and giving praises)}

Benevolent speeches are assertive sentences expressed in working contexts by which an individual displays positive and warm attitudes toward women while cognitively regard female workers as incompetents. The author terms this type of speech based on the definition of Benevolent Sexism (BS) in psychology [1], and here are two subcategories of it. One subcategory is speeches showing warmth toward women by covertly viewing women based on traditional stereotypes and restricted social roles [1]. The other is directly giving praises which are specifically referred to positive comments on a woman about a certain property which is regarded as an essence of the membership in the social category of women. The author categorizes these two speech acts into the same group because both of them show a more positive attitude overtly but stereotype the social group of women covertly and have detrimental effects on women's performance (which will be discussed in the next section). Examples are shown in Table 2.

Table 2 Examples for Benevolent Speeches

1. Mary will face great difficulty in our construction industry, and we should assign some easy tasks to her.

2. It is uneasy for Susie to work and live alone here, and we should take care of her.

3. You did a great job. Girls are indeed attentive.

4. You are suitable for a sales position because women are compassionate.

S1-2 are unjustified expectations of women generalized by stereotypes such as "women are delicate." and "women are innocent." Specifically, S1 displays a benevolent attitude to the woman named Mary literally by proposing that women should be assigned more "easier" and "manageable" tasks for them. Nevertheless, the speaker implicates that the woman he is talking about is not as competent as men in the construction industry, which is normally regarded as a field dominated by male workers. S3-4 are assertive sentences of direct praises for women's good performances of a specific task or in a particular field. No matter how positive the speeches are, this type of speech makes essentialists beliefs of the social category of women by blatantly linking their gender to an essentialized features such as being sensitive, attentive and compassionate. These features indeed generate from traditionally stereotyped female gender roles, most of which are characteristics of domestic roles [18]. It is worth noting that these restricted female properties might ultimately put women in a minimal scope of occupation choices [4]. Praises falling into this category fail to acknowledge women's individualism and thus ignore their individual property in terms of women' $s$ occupational choices.

\section{NEGATIVE EFFECTS OF THE TWO TYPES OF SPEECH}

In this section, on the basis of the analysis of gender-biased stereotypes embedded in the two types of speech in varied occupational contexts, the effects the stereotypes potentially have on the career development of women will be discussed in this section.

One of the effects is that the stereotypes about women embedded in the speeches in the workplace, which take the form of generic generalization, are likely to reduce career opportunities for women and undermine occupational gender equality. Cognitively, essentialist beliefs in people' $\mathrm{s}$ mind are more central to stereotype structure [26], and the essentialized features can be transmitted through stereotypes [8]. As a result, people tend to use essentialist beliefs, an easier cognitive tool, to make explanations and expectations about the features of others. Therefore, if the gender stereotypes discussed previously are made prevalently 
and in public, the prejudice toward women will be transmitted and reinforced and thus, damages are probably done for women's career development.

These reinforced stereotypes of women might decrease their career opportunities if more and more employers endorse these essentialist beliefs about women. Suppose that in a job interview in the profession of accounting, there are a female and a male candidate with the same qualities for the position except for their gender. Conditionally, the interviewer firmly holds a set of essentialist beliefs to judge the female candidate based on her membership of the social category of women, not on her individuality. The interviewer would get access to the stereotype in his mind that "men are better at math than women" and thus, believe this is a fundamental discrepancy between men and women. Consequently, the female is deprived of the job opportunity only because of her gender.

Furthermore, stereotypes expressed in generic language is detrimental to women's conceptions of their occupational abilities. Generic generalizations trigger entity mindset, which means people tend to view skills as fixed, stable, and cannot be developed, whereas people with incremental mindset believe that skills can be improved by learning and practice [8]. It is highly likely that females who are questioned and commented on by the stereotyped speeches in different work contexts would endorse the entity theory with their working abilities. The author argues that entity theoretical thinking will affect women' s conception of working abilities from two aspects. Firstly, women might lack motivations and give up opportunities to improve their capacity and expand their career paths under the conception that individuals' abilities are inherent and therefore, unchangeable. Secondly, entity mindset would render women distressed when dealing with failure in work, as they tend to regard failure in one task as evidence for their permanent incompetence, especially in terms of the tasks in masculine domains. Consequently, they would retreat from those domains and choose to develop their career in fields categorized stereotypically as "feminine domains" .

In particular, this study wants to point out what effects that benevolent speeches and praises have on women's career development. Leslie and Dardenne et al. make the claim that positive stereotypes (if activated directly) and Benevolent Sexism trigger women' $s$ doubt of their competence [8][22]. This is partly because women hearing stereotyped sentences might be suspicious of their capacity of the entity and feel inferior. The negative effects of benevolent speeches and praises are subtle and unspoken. However, given their impairment to performances and competence, they should be made salient.

\section{CONCLUSION}

Speeches embedded with gender stereotypes in occupational contexts toward women is a frequent enough occurrence to attract theorists and empiricists to do further research in different cultures. The current study, through the analysis of examples of speech in occupational contexts, has displayed what and how stereotypes are expressed in different professions across cultural and social settings. Based on the discussion, the author has argued that they are likely to reduce career opportunities for women by reinforcing the stereotypes of women and to impair their work performances and competence. Stereotype is a major contributor to prejudice in society. However, they are expressed in too implicit and unconscious ways for both speakers and recipients to perceive.

Eagly and Mladinic show evidence that women may have gained more positive and favorable stereotypes and evaluations in a broader social context [18]. Nevertheless, women still face many barriers in obtaining working opportunities, being paid equally as their male counterparts, and being entitled to hold structural powers in their professional arenas, at least in the culture where the author of this paper comes from.

Therefore, this study offers a way to understand stereotypes of women in occupational spheres more and reminds people to keep conscious of stereotypes in their minds and speeches. In the future, a larger size of examples with more variants of stereotyped speeches toward women in working contexts, and even in a wider social setting, should be explored. Thus, this kind of discussion might be able to raise the public awareness of the gender-biased stereotypes in their minds and expressions. Only on the basis of widespread awareness would solutions to reduce the detrimental effects in question become plausible.

\section{REFERENCES}

[1] Glick, P., \& Fiske, S. T. (1996). The ambivalent sexism inventory: Differentiating hostile and benevolent sexism. Journal of personality and social psychology, 70(3), 491.

[2] Beeghly, E. (2015). What is a stereotype? What is stereotyping? Hypatia, 30(4), 675-691.

[3] Haslanger, S. (2011). Ideology, generics, and common ground. In Feminist metaphysics (pp. 179-207). Springer, Dordrecht.

[4] Hideg, I., \& Ferris, D. L. (2016). The compassionate sexist? How benevolent sexism promotes and undermines gender equality in the workplace. Journal of Personality and Social Psychology, 111(5), 706. 
[5] Lawler, J. M. (1973). Studies in English generics. University of Michigan.

[6] Carlson, G. N. (1977). Reference to kinds in English. University of Massachusetts Amherst.

[7] Leslie, S. J. (2007). Generics and the structure of the mind. Philosophical perspectives, 21, 375-403.

[8] Leslie, S. J. (2014). Carving up the social world with generics. Oxford studies in experimental philosophy, 1.

[9] Pelletier, F. J., \& Asher, N. (1997). Generics and defaults. In Handbook of logic and language (pp. 1125-1177). North-Holland.

[10] Prentice, D. A., \& Miller, D. T. (2006). Essentializing differences between women and men. Psychological Science, 17(2), 129-135.

[11] Fiske, S. T., \& Taylor, S. E. (1991). Social cognition. Mcgraw-Hill Book Company.

[12] Haslam, N., Rothschild, L., \& Ernst, D. (2002). Are essentialist beliefs associated with prejudice? British journal of social psychology, 41(1), 87-100.

[13] Allport, G. W., Clark, K., \& Pettigrew, T. (1954). The nature of prejudice.

[14] Billig, M. (1985). Prejudice, categorization and particularization: From a perceptual to a rhetorical approach. European Journal of Social Psychology, 15(1), 79-103.

[15] Eagly, A. H., \& Mladinic, A. (1994). Are people prejudiced against women? Some answers from research on attitudes, gender stereotypes, and judgments of competence. European review of social psychology, 5(1), 1-35.

[16] Blum, L. (2004). Stereotypes and stereotyping: A moral analysis. Philosophical papers, 33(3), 251-289.

[17] Glick, P., \& Fiske, S. T. (1997). Hostile and benevolent sexism: Measuring ambivalent sexist attitudes toward women. Psychology of women quarterly, 21(1), 119-135.

[18] Glick, P., \& Fiske, S. T. (2001). An ambivalent alliance: Hostile and benevolent sexism as complementary justifications for gender inequality. American psychologist, 56(2), 109.

[19] Dardenne, B., Dumont, M., \& Bollier, T. (2007). Insidious dangers of benevolent sexism: consequences for women's performance. Journal of personality and social psychology, 93(5), 764.

[20] Dumont, M., Sarlet, M., \& Dardenne, B. (2010). Be too kind to a woman, she'll feel incompetent: Benevolent sexism shifts self-construal and autobiographical memories toward incompetence. Sex Roles, 62(7-8), 545-553.

[21] von Hippel, C., Sekaquaptewa, D., \& McFarlane, M. (2015). Stereotype threat among women in finance: Negative effects on identity, workplace well-being, and recruiting. Psychology of Women Quarterly, 39(3), 405-414.

[22] Barreto, M., \& Ellemers, N. (2005). The burden of benevolent sexism: How it contributes to the maintenance of gender inequalities. European journal of social psychology, 35(5), 633-642.

[23] Hammond, M. D., \& Cimpian, A. (2017). Investigating the cognitive structure of stereotypes: Generic beliefs about groups predict social judgments better than statistical beliefs. Journal of Experimental Psychology: General, 146(5), 607. 